\title{
Bridging the Gap of Idea Management Systems Application and Organizational Effectiveness with Adaptive Structuration Theory
}

\author{
Elina Mikelsone ${ }^{1}$, Elita Liela $^{2}$ \\ Management Department, BA School of Business and Finance, Riga, Latvia \\ E-mails: ${ }^{1}$ mikelsone.elina@gmail.com (corresponding author); ${ }^{2}$ elita.liela@ba.lv
}

Received 24 February 2017; accepted 24 March 2017

\begin{abstract}
Information technologies that help to manage knowledge have a scientific and practical topicality. Among such technologies are web-based idea management systems (IMS). But there is little scientific evidence on how web-based IMS application materializes with in organizations and how they relate with organization effectiveness (OE). Authors of this paper aim to develop conceptual bases to fill this gap. The paper is based on an extensive review of literature about IMS, OE, and Adaptive Structuration Theory (AST). The aim is to develop a new research framework to explore how can the concepts IMS, AST, OE, - be operationalized for empirical research to explore how IMS application and its results relate with OE and what are the main input and process elements in these relations? Based on a systematic literature review, that was analysed through content analysis technique and exploratory meta-analysis, authors created the adapted framework of AST for IMS context and proposed hypothesis. The paper contributes by developing detailed characterization of AST construct in webbased IMS application context on organizations. Authors propose theoretical guidance on how to explore the IMS impact on $\mathrm{OE}$ and expands the domain of outcomes of AST researching outcomes of web-based IMS by including OE dimensions.
\end{abstract}

Keywords: adaptive structuration theory, idea management systems, organizational effectiveness, web-based idea management systems, information technologies, literature review.

JEL Classification: M190, M150, M110.

Conference topic: Informational Society: Challenges, Solutions and Tendencies.

\section{Introduction}

The ongoing changes in the world have made the use of knowledge and information technologies for the greatest good a clear priority (INSEAD 2016). There is a scientific and practical topicality of information technologies that helps to manage knowledge and information. There are different kinds of information management systems, such as, electronic messaging systems, collaborative systems, group decision support systems, etc. One of such tools are web-based idea management systems (IMS). In previously conducted research (Mikelsone, Liela 2015) authors have developed definition of IMS based on more than 70 literature sources. Definition developed during the research is that IMS are systematic, manageable tools, tool kits that helps users to generate, evaluate and continuation of this process. First it is important to research web-based IMS, because it coincide with global tendencies. Three tendencies that authors think will increase web-based IMS importance: (1) in the age of knowledge based economy tools that provide means for acquiring, evaluation and development of knowledge and ideas are extremely important; (2) the growing role of ICT increases the importance of web-based tools that allow to support innovation process; (3) web-based IMS are becoming more important in the context of open innovation and co-innovation, giving the access to both internal and external sources of ideas and knowledge.

Second, the use of web-based IMS has become a part of organization culture. But although there is a great variety of web-based IMS and as shown previously many well-known organizations used them, the situation that was described in legendary publication "Capturing the Complexity in Advanced Technology Use: Adaptive Structuration Theory" by DeSanctis and Poole (1994) is still topical: developers and users of information management systems see potential of these systems but positive outcomes often do not occur inconsistently and that is one of the reason why organizations do not use them widely. Authors believe that the use of web-based IMS can have a significant impact on organization effectiveness (OE) both good and bad. Although the concept of OE is encountered repeatedly in the organizational literature, but there is only a rudimentary understanding of what is involved in the concept. That is the reason why authors conducted (Mikelsone, Liela 2016) additional research to define the OE term based on more than 130 peerreviewed publications about OE. This definition will be used as one of the conceptualizations and it defines OE as a

(C) 2017 E. Mikelsone, E. Liela. Published by VGTU Press. This is an open-access article distributed under the terms of the Creative Commons Attribution (CC BY 4.0) License, which permits unrestricted use, distribution, and reproduction in any medium, provided the original author and source are credited. 

adaptive structuration theory

multidimensional measurement which could consist form financial/nonfinancial, internal/external, subjective and objective dimensions, which reflects achievements of the organization, but the dimensions of OE could be different in different contexts.

Third, many researchers concentrate not only to explore different information technologies but also their application and impact. And according to basic scanning of 7 data bases there are more than 100 papers, proceedings and notes that aim to explain it. There are two main trends in the literature. First, to explore overall IT management and use different aspects, for example, malleable IT use, (e.g. Schmitz et al. 2016), technology adaption in groups (e.g. Saskia-Bayerl et al. 2016), information system project control (e.g. Wiener et al. 2016), social inclusion and IT (e.g. Diaz Andrade, Doolin 2016), ICT and wellbeing of nations (e.g. Ganju et al. 2016), IT impact on the performance of companies (e.g. Sabherwal, Jeyaraj 2015), ICT for development (e.g. Lin, Myers 2015), IT use as learning mechanism (e.g. Iyengar et al. 2015), etc. The aim of these researches is to explore specific aspects of ICT in general. Second trend - to explore specific type of IT, for example, group support systems (e.g. Lolli et al. 2015; Bose 2015), crowdsourcing (e.g. Deng et al. 2016), gaming platforms (e.g. Ketter et al. 2016), knowledge management systems (Hyun-Kim et al. 2016), sponsored search platforms (Im et al. 2016) etc. The aim of these researches is to research specific ICT types and related aspects. In this paper authors use the second construct- exploring the specific aspect related with specific ICT because it is more focused view on the issue. Growing number of publications about IM shows that there is interest in academic community about IM, but still there is a lack of academic research papers about many crucial aspects of IM. IM and IMS have been researched by many researchers (e.g. Bjork, Magnusson 2009; Barczak et al. 2009; Westerski 2013; Summa 2004; Bakker et al. 2006; Gamlin et al. 2007) and the acquired results tend to be similar emphasizing the potential of IM and IMS. Mostly researchers research IM and its features but less about IMS and researches about web-based IMS are rare in scientific literature. Such quantity of researches forms theoretical basis for future research. But it must be noted that there is little theoretical guidance as to how application of IM and IMS materialize within organizations and even less direction on how to conceptualize and examine the effects of IM and its systems on OE. One of the main research gaps in the literature is: how application of web-based IMS relates with OE and how to increase OE with IMS application. This research gap was verified by the authors in previous researches (Mikelsone, Liela 2016).

Fourth, the absence of an established theoretical framework that anchors IM and OE necessitates an examination of peer-reviewed journals, books, proceedings that are published in the main scientific databases. With references, Adaptive Structuration Theory (AST), this study presents an overview of OE and IMS literature in connections with AST and discusses noteworthy research hypothesis that may guide forthcoming empirical and theoretical investigation. Conceptually, the papers draw on Poole and DeSanctis concept of Adaptive Structuration Theory. And relevant amendments to the concept as well as empirical evidence from the studies exploring aspects of IM, IMS and OE. According to the DeSanctis and Poole (1994) established adaptive structuration theory information technologies do not automatically improve effectiveness (Kessler 2013) and it gives the basic framework on how to research ICT's impacts on organization and its outcomes depending on the structures, incorporated technology, and other structures that emerge as users attempt to adapt these technologies.

Aim of the research is to develop general research framework to explore how can the concepts - IMS, AST and OE, - operationalized for empirical research to explore how IMS application and its results relates with OE.

To fill the gap authors apply theoretical approach, literature review, with the main aim to develop theoretical framework on how to research it. Parallelly conducting exploratory meta-analysis to develop hypothesis.

Paper also aims to answer to the research questions: (RQ1) what is meant by IMS and OE in AST context in conceptual level; (RQ2) what are the main input, process and outcomes of web-based IMS application; (RQ3) what are the main hypothesis to explore how IMS within AST could impact OE? The answer to these questions implies an answer to the underlying composite question: how can the concepts of IMS, AST and OE operationalized for empirical research to explore how IMS application and its results relates with OE?

There are several contributions: (1) characterized AST construct in IMS context; (2) research framework to research AST not focusing on groups or individuals, but on organizations; (3) aim to create theoretical framework - this paper fulfils an identified need to explore how to increase OE by web-based IMS application - by giving theoretical guidance on how to explore the IMS impact on OE; (4) authors expand the domain of outcomes of AST research by including OE dimensions that are normally found in the OE literature. So, providing theoretical idea how to research how IMS outcomes- effectiveness correlates with OE.

Research object - in literature based framework of IMS and OE within AST. Research tasks: (1) research literature about OE, IMS and AST; (2) create general research framework and hypothesis for web-based IMS and OE research in AST context. Research methodology - systematic literature review analysed with content analysis and qualitative meta analysis.

The rest of the paper is structured as follows. The second section introduce research background, while section 3 discusses methodology. Section 4 continues by presenting the answers to the research questions through conducted research, based on that authors create theoretical framework for future researches and presents potential hypothesis The main conclusions are covered in the last chapter. 


\section{Background}

In this section authors based on the basic literature review overviews the main concepts of the paper. With the aim to answer to the first research question - what is meant by IMS and OE in AST context in conceptual level.

\section{Adaptive structuration theory}

The theoretical ground of AST could be found on structuration theory that was proposed by Giddens (1984) and based on the idea that structural properties depends upon its use and exists through human - agent activities. Adaptive Structuration Theory developed by Poole and DeSanctis (1990) based on the idea that application of information technologies does not automatically improve outputs, but it depends on its appropriation by users. AST originally was applied to study group decision systems (e.g. DeSanctis, Poole 1994; Gopal et al. 1992), so focusing on group level. But in recent decade there are some researches that study systems in individual (e.g. Schmitz et al. 2016; Wang et al. 2016), and organization level mainly concentrating on specific ICT and non-ICT processes and aspects (e.g. Edgington et al. 2010). This theory has been approved by the studies to be useful to study also non-ICT topics, like supply chain and process strategy, virtual teams, industrial marketing etc. (e.g. Droge et al. 2012; Kirkman, Mathieu 2005).

One of AST creators Poole (2013) explains that AST posits that systems, such as groups and organizations, can be explained in terms of their activity in structures and structuration is the production and reproduction of system through members' appropriation of rules and resources. Overall AST argues that the effects of ICT on outcomes depend on the ICT structures and on the adaptive structures that forms when member apply ICT. Structures and features are produced and reproduced through structuration process that occur as system appropriates them in ongoing activities. Structuration process also is influenced by external structures, like, tasks, rules and culture. Structure and system concept overlapping see in Figure 1.

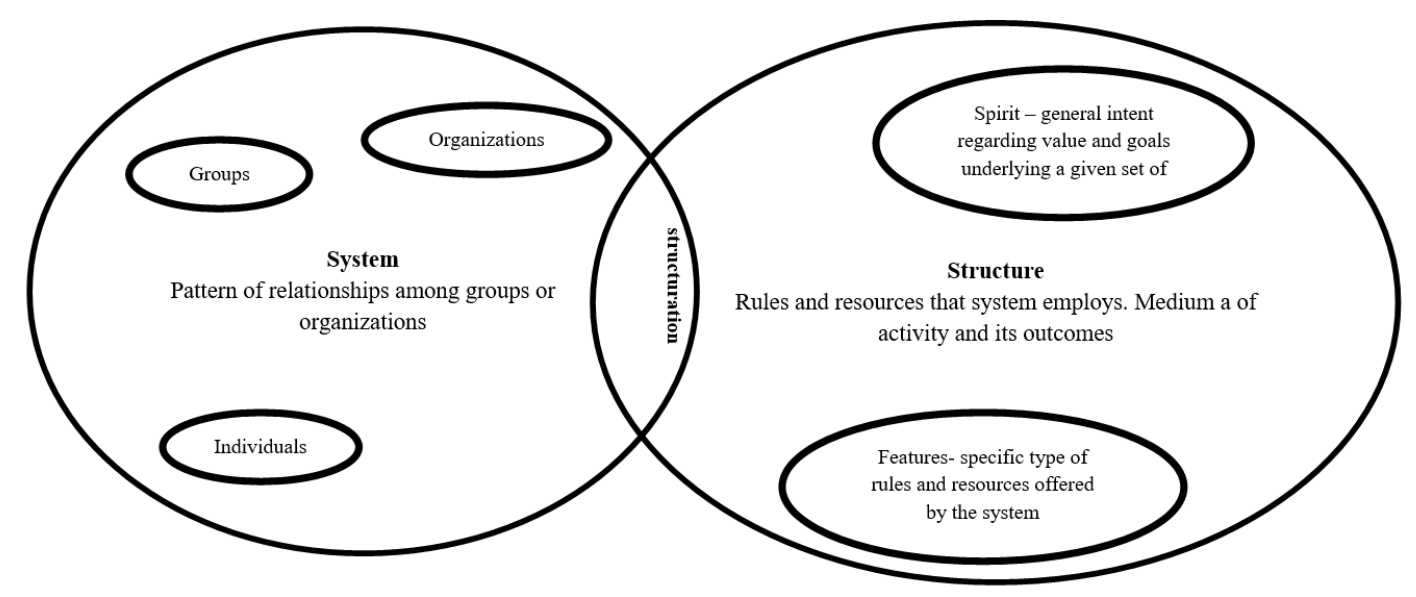

Fig. 1. Systems ans Structures in AST (Source: compiled by authors)

According to Poole (2013) several sets of factors influence the structuring process - characteristics of the ICT (restrictiveness, sophistication, standardization complexity); task characteristics (difficulty and complexity) and characteristics of the systems environment (dynamism and hostility); characteristics of internal system of organization (culture, norms, leadership). These three sets of factors are sources of structure, but the system is directly influenced only by those elements which are incorporated in activities. Outcomes according to AST could be different in each case, for example, effectiveness, efficiency, new structures, commitment, learning and cohesion etc. But even as there is one common AST model, every research adapts it for the specific research context. It is vitally important to adapt it appropriately. So RQ1-what is meant by IMS, AST and OE in conceptual level - will be basis to create appropriate adoption of the model.

From practical perspective, AST helped to develop new knowledge about ICT implementations and related aspects. For example, Lee (2012) presents a new method for group decision making with incomplete fuzzy preference relations based on the additive consistency and the order consistency, Mutch (2002) offers more productive way of approaching information systems, Kang and Santhanam (2003/2004) proposed framework calls for trainers to be continuously engaged with users and help refine their knowledge during the process of appropriation, Reinig and Shin (2002) explored that group support systems are effective in reducing process losses etc. A key skill for ICT management is learning to read the process of adaptive structuration so that they can channel it in productive directions (Poole 2013). AST has been criticized by some for being overly "positivistic" and that it does not provide any usable methodology. But there are many studies that bridge quantitative and qualitative approaches in inquiry and emphasize a prior construct definition and attention to validity of measurement. There are new methodologies applied for AST - 

adaptive structuration theory

laboratory experiments, structural equation modelling, and critical analysis have been conducted utilizing AST, providing additional verification for validity of this theory. The basic model of AST that is applied in this research see in Figure 2.

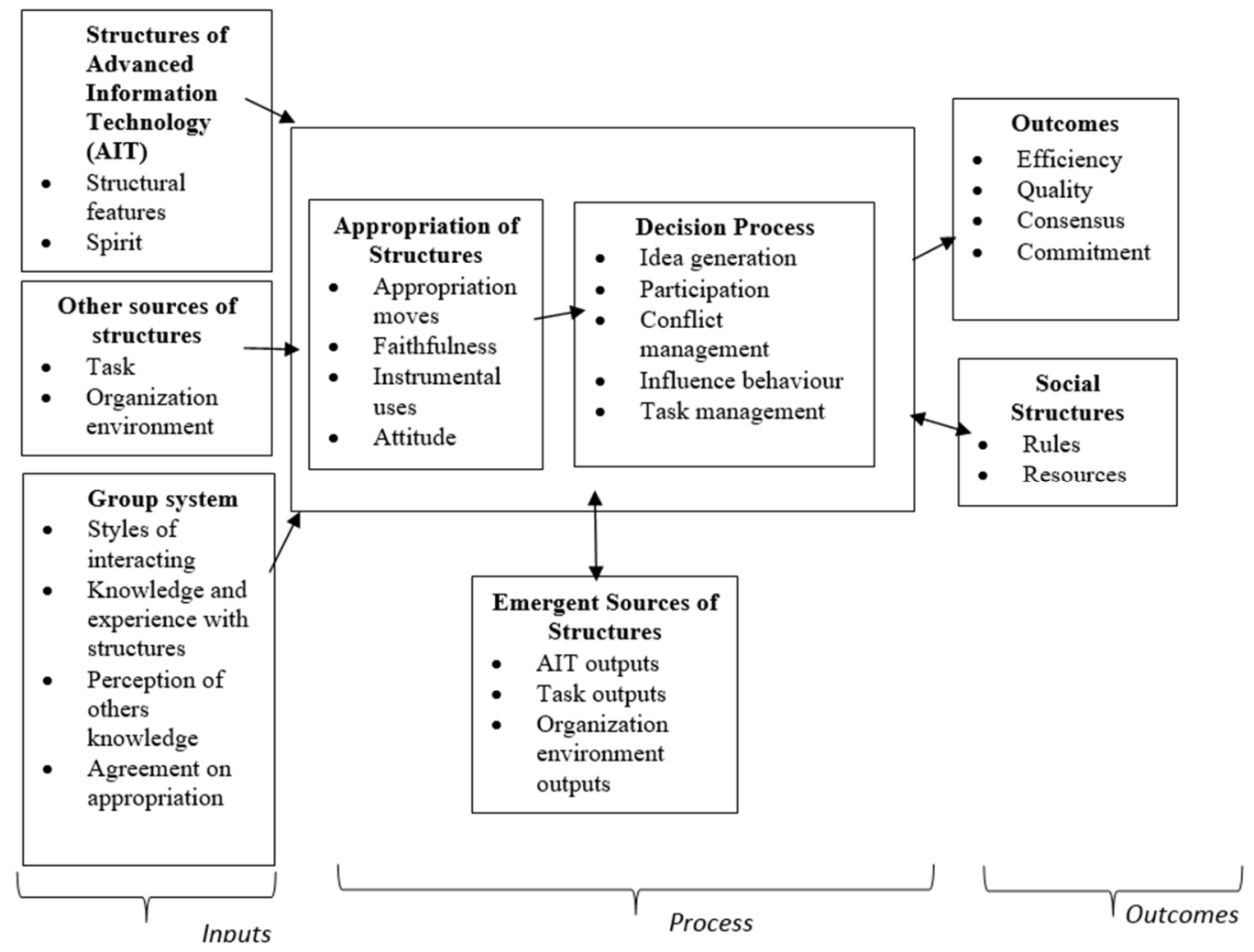

Fig. 2. Summary AST Constructs (Source: DeSanctis, Poole 1994)

\section{Idea management systems}

In IMS research not only direct literature about IMS is important but also about IM. First, because some researchers do not separate terms IM and IMS. In this paper authors define IM as systematic, manageable process of idea generation, evaluation and development, but IMS tool, tool kit or complex system which provides systematic, manageable process of idea generation, evaluation and development (Mikelsone, Liela 2016).

Second, IM literature gives wider ensign in IMS concept, exploring more social (e.g. Selart, Johansen 2011; Vandenbosch et al. 2006) and more structural elements of IM (e.g. Deichmann 2012; Westerski 2013). In this paper both factors are important so all literature will be overviewed. There is also a trend in IMS literature that mostly existing IMS and their application and potential improvements are researched (e.g. Westerski et al. 2013; Bertetta 2015), but some researches also aims to research development of new IMS and related issues (e.g. Applegate 1986; Bothos et al. 2012; Lowe, Heller 2014). This paper aims to be part of the first type of papers which explores how to apply IMS, in this case- how to apply them more effective and what influences these processes. Additional research in Google Scholar and Scopus represents that the most cited authors about IM are Bothos, Westerski, Vandenbosch, Brem, Flyn, Sandstrom. In this paper not only main authors researches will be overviewed but also newest literature. In this paper author concentrates only on web-based IMS, based on the leading trends.

It should be noticed that there are researches (e.g. Reinig, Shin 2002; Dennis, Garfield 2003; Lee, Zo 2016) which have explored group decision support with the help of AST and structuration theory. That shows that these theories could be applicable to explore IMS application. But even as there are many researches which explore IM and IMS there is a research gap, because there are no researches that gather information to answer the question -what are the main input factors, process and outcomes of web-based IMS application?

\section{Organizational effectiveness}

OE is one of the main topics in management for many decades (Goodman, Saks 1977; Biswas 2010) and in recent decades topicality has even grown (Mausolff, Spence 2008; Lecy et al. 2012), but there is still no one view on its concept. One of the oldest and widest approaches is that OE is multidimensional measurement (Angle, Perry 1981; 

adaptive structuration theory

Dension 1990). There are different kind of OE models, for example, Goal Attained Model, Functional Model, Competing Values Model, Systems (Resource) Model, Open Systems Model, Reputational approach, Internal Process Model, HR Model. It shows that measurements of OE could differ and mostly they are multidimensional. Authors (Mikelsone, Liela 2016) have gathered 199 possible OE dimensions that are used in OE researches. These dimensions could be applied to research OE, but the selection of them depends on context and research aim. That is reason why in this research authors use only general concept of OE not several dimensions. Because the aim is to create general model, not specified.

Based on the multidimensional and complex nature (specific to context) of the OE, authors in this research as basic concept of $\mathrm{OE}$ will apply definitions, that $\mathrm{OE}$ is "a multidimensional measurement that could consist form financial/nonfinancial, internal/external, subjective and objective dimensions, which reflects achievements of the organization, but the dimensions of OE could be different in different contexts (Mikelsone, Liela 2016)".

\section{AST and OE in IMS application context}

There are no researches that directly explore IMS and OE relations, but there are many researches about IMS, AST and OE which could be used as a basis for this paper. Authors see opportunity to research how web-based IMS application and OE relates within application of adapted adaptive structuration theory. So the main question is how can the concepts of IMS, AST and OE operationalized for empirical research to explore how IMS application and its results relates with OE? To answer this question with creation of the research framework and hypothesis it is important to answer to the several additional questions - (RQ1) what is meant by IMS and OE in AST context in conceptual level; (RQ2) what are the main input, process and outcomes of IMS application; (RQ3) what are the main hypothesis to explore how IMS within AST could impact OE? In this section the main concepts are described so developing the basics for the RQ1 answer in the further paper this question will be described in detail in the AST context. The next Section of the paper describes the methodology how authors will seek the answer to these questions and will create framework and general hypothesis.

\section{Methodology}

Theoretical framework development is based on a theoretical research method - critical literature review. Data collection was conducted in 4 stages: (1) to research scientific databases to explore literature where terms "idea management", "organisational effectiveness", "adaptive structuration theory" are mentioned, every term was researched in 7 databases; (2) to select literature directly about IM, OE, AST; (3) to exclude duplicates; (4) to apply selected literature. At the first research stage 4283216 literature sources in which "idea management", 1029401 - "organisational effectiveness" and "adaptive structuration theory" - 22584 was mentioned were found. At the second research stage literature was selected literature were these terms mentioned. In third stage duplicates were excluded. 75 - IM, 133 - OE, 111 - AST literature sources passed the third stage. Detailed literature source count in different stages is reflected in Table 1.

Table 1. Literature selection by stages (Source: compiled by authors)

\begin{tabular}{|c|c|c|c|c|c|c|c|c|c|c|}
\hline & \multicolumn{3}{|c|}{$\begin{array}{l}\text { Stage } 1-\text { in article title or/and } \\
\text { keywords mentioned terms: }\end{array}$} & \multicolumn{3}{|c|}{$\begin{array}{l}\text { Stage } 2 \text { - directly about } \\
\text { (full text available): }\end{array}$} & \multicolumn{3}{|c|}{$\begin{array}{l}\text { Stage } 3 \text { - unique } \\
\text { sources: }\end{array}$} & $\begin{array}{l}\text { Stage } 4-\text { for } \\
\text { meta analysis }\end{array}$ \\
\hline & IM & $\mathrm{OE}$ & AST & IM & $\mathrm{OE}$ & AST & $\mathrm{IM}$ & $\mathrm{OE}$ & AST & \multirow[t]{11}{*}{195} \\
\hline Scopus & 39702 & 16592 & - & 15 & 36 & - & \multirow[t]{10}{*}{82} & \multirow[t]{10}{*}{133} & \multirow[t]{10}{*}{109} & \\
\hline ScienceDirect & 364611 & 78381 & 521 & 2 & 24 & 15 & & & & \\
\hline Google Scholar & 3980000 & 23700 & 18300 & 33 & 15 & 11 & & & & \\
\hline Sage Journals & 152934 & 54575 & 590 & 8 & 34 & 63 & & & & \\
\hline Ebsco & 5129835 & 832645 & 316 & 9 & 33 & 17 & & & & \\
\hline Emerald & 107825 & 23456 & 445 & 3 & 25 & 9 & & & & \\
\hline Web of Science & 269 & 52 & & 52 & 8 & & & & & \\
\hline Jstor & - & - & 755 & - & - & 26 & & & & \\
\hline Taylor \& Francis & - & - & 1647 & - & - & 2 & & & & \\
\hline Sum: & 4645341 & 1029401 & 22584 & 122 & 175 & & & & & \\
\hline
\end{tabular}

All the sources that passed the stage 3 were conducted in systematic literature analysis to analyze overall research trends. Systematic literature analysis was conducted in 3 step process. First, to make the research process more effective, before content analysis a review protocol was developed. The development of a protocol is essential to codify as precisely as possible the way studies have been collected to answer a specific research questions, namely: (RQ1) what 

adaptive structuration theory

is meant by IMS and OE in AST context in conceptual level; (RQ2) what are the main inputs, processes, and outcomes of IMS application; (RQ3) what are the main hypothesis to explore to research how IMS within AST could impact OE? Second step - data extraction. Third step - information synthesis with the help of thematic analysis. This methodology is developed based on Boiral (2012) applied methodology but with improved third step - conducting information synthesis more systematic with the help of content analysis. That kind of approach was applied based on the research type and aim. Content analysis is technique that helps to identify and analyze qualitative data. This method is a descriptive method and it can be applied through both inductive and deductive approaches. Content analysis instead of thematic analysis was selected because this technique provides not only qualitative, but also quantitative insights which in this case are important to explore the most frequent applied IMS, AST, and OE dimensions.

In this research on the most frequent applied content analysis model was applied. Model consisted from 3 steps (based on Vaismoradi et al. 2013): (1) preparation - in this step all developed literature source analysis protocols were overviewed; (2) organization - initial coding was conducted nonlinearly, there was predefined codes and codes that were data driven. In this step all data from protocols were coded and categorized. Were created also 3 level category network with global, organizing and basic categories. In this Phase were also developed basic descriptions of these categories based on the data; (3) report development - described categories that are important for the research and developed answers to the research questions.

Developed theoretical framework and hypothesis were verified and supported by literature sources selected in stage 4 . That is - selected only dissertations, scientific institute researches and 2-4* ABS ranking included journal articles. With the stage 4 selected literature were conducted exploratory meta - analysis to present hypothesis for further examination. The aim of meta-analysis is to conduct the selected literature additional research in structural and objective manner, by extracting variables and relationships from the studies, and analyzing these data (Hooff et al. 2005). This paper applied exploratory approach of meta-analysis. To establish the relevant variables and relationships in this area, resulting evidence of support for hypothesis and hence further examination (Hooff et al. 2005). This approach consists from three steps: identification of the literature (this step was done with literature review), development of concept-centric matrix based on the proposed theoretical framework, third- assessment of the literature. The next Section of the paper deals with development of theoretical framework for future researches by answering proposed RQ and developing general hypothesis for future researches.

\section{Created theoretical framework and hypothesis}

AST is considered an appropriate theoretical framework for this study, because it examines not only organizational changes facilitated by different types of structures, but also could reveal how ICT tools influence process and its outcomes. AST posits that the way technology is applied by an organization is determined by several interacting forces: the technology, the organization's environment, the task, and the way structures emerge and update throughout the appropriation process (Desanctis, Poole 1994; Ajjan et al. 2016). Even at the beginning AST has been applied to study the adaptation of GSS, it can be used in the context of a broader set of advanced information technologies (Desanctis, Poole 1994; Dennis, Garfield 2003), such as web-based IMS. This paper takes the view that implementations of webbased IMS are social, technical and structured by their contexts of implementation and application. Although AST provides the framework to understand the interaction between individuals, groups, and technology, there is little evidence on how specific ICT tool application within the organizational environment may impact outcomes associated with structures and appropriation of it (Charlier et al. 2016). That is the reason why in this study the main focus is on organizational level research web-based IMS application within AST framework.

AST is employed as the basic framework to present hypotheses in this study. A total of three main constructs (inputs, outputs, process) with seven sub-constructs (structures of AIT (in this paper web-based IMS), other sources of structures, group (in this paper organization) system, social interaction (appropriation of technology, decision making process (in this paper IM process in web-based IMS)), emergent sources of structures, outcomes, new social structures) are discussed in AST. These constructs are used to describe how web-based IMS application in organizations influences OE.

\section{Inputs}

AST focuses on structures provided by technologies, processes, and organizations (DeSanctis, Poole 1994). Past literature has considered two sources of structures: artefacts (e.g. technologies, tasks and processes) social actions of the participants and their context (DeSanctis, Poole 1994; Ajjan et al. 2016). There are 3 main input attributes structures of AIT, other sources of structures, organization system. In this paper, AIT is defined as web-based IMS, and the structural features of web-based IMS are referred to as 'web-based IMS technology features'. Structures of technology according to AST are characterized by features provided by the system. The essence of technology refers to the main intent with regard to the values and goals underlying a given set of technology features. As indicated in previous studies, the features of web-based IMS - idea generation feature, idea evaluation feature, parallelism, anonymity, idea retaining etc. - are compliant with the structural features. The values underlying these structural features 

adaptive structuration theory

make web-based IMS a new AIT, which differs from innovation management systems, design thinking systems, group decision making systems.

Based on the AST literature authors provide hypothesis: H1 Web-based IMS features have a positive influence on web-based IM process and its outcomes. Qualitative meta-analysis supports this hypothesis. Some of possible variables described by researchers as 'web-based IMS technology features' are idea generation (Summa 2004), idea evaluation (Westerski 2013; Summa 2004), idea retention (Dennis, Garfield 2003; Summa 2004), parallelism and anonymity (Dennis, Garfield 2003), external and internal IM (Summa 2004; Bertetta 2015). These variables could be applied to create detailed hypothesis for future researches.

The main ideas and hypothesis see in Figure 3.

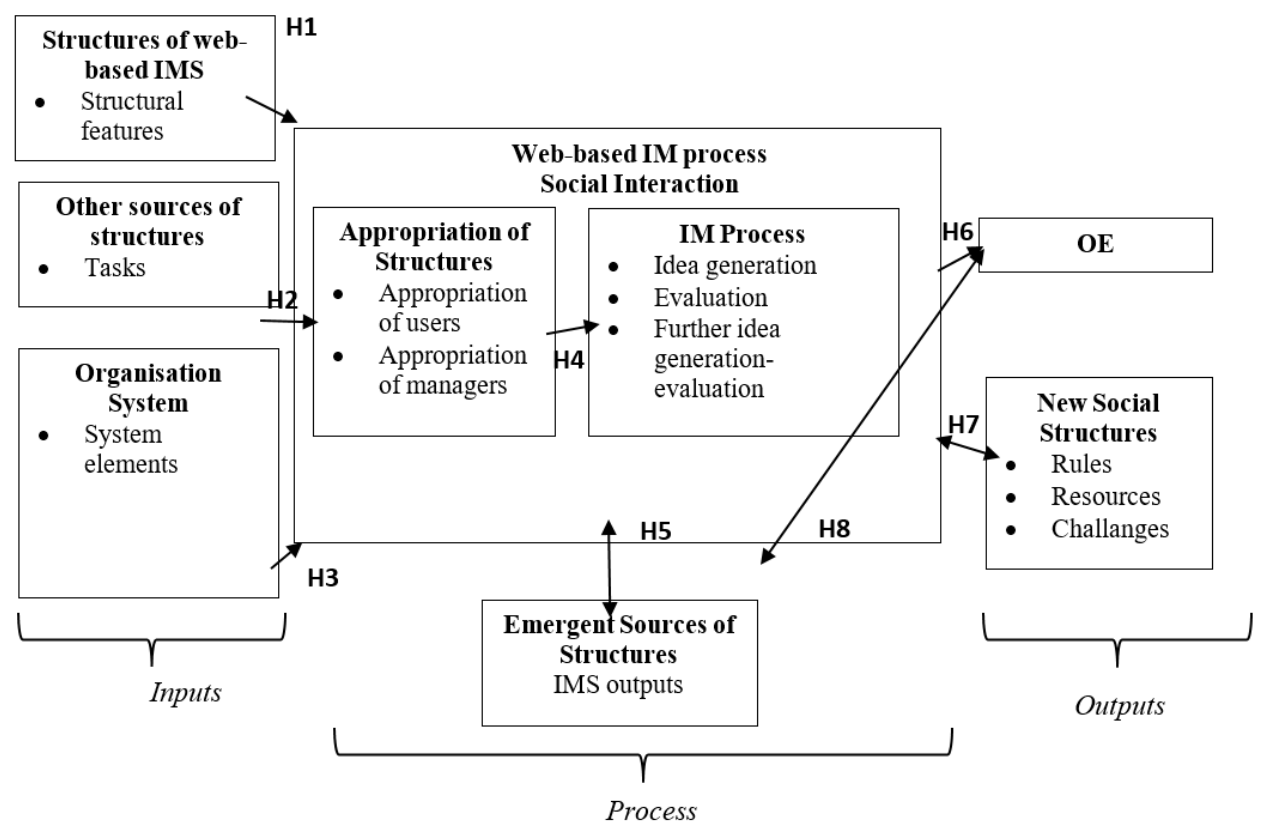

Fig. 3. General AST Framework for Web-Based IMS researches (Source: compiled by authors)

As described in the background, main parts of idea management are idea generation, evaluation, and retention of ideas. It is important to research impact of them on web-based IMS application process. As one part of idea management is further idea generation and evaluation, idea retention feature becomes important. Researched studies also revealed other interesting features, such as, parallelism - ability for members to exchange information simultaneously (Dennis, Garfield 2003). That means that users can generate and evaluate ideas at the same time. Anonymity is stressed as important feature. That enables members to make contributions without attaching their names which is not possible when contributions are made verbally (Dennis, Garfield 2003). Summa (2004) has stressed out that IMS provides transparent IM process and feedback that improve the quality and number of new ideas. That study has mentioned also other functionalities that could be researched as features, such as: (1) support for centralized and decentralized IM; (2) support for electronic attachment; (3) search capabilities; (4) ability to integrate with other systems; (5) user and group management; (6) collaboration; (7) enable both predefined and ad-hoc reporting; (8) idea classification. Theoretical approach does not provide the full understanding of web-based IMS technology features. Authors suggest for future researches conduct additional empirical research to choose the technology features to include in the research. There could be three advisable approaches: (1) based on the theory proposed technology features to use expert method; (2) conduct technology research by gathering the most frequent applied features; (3) to mix both approaches.

Other sources of structure show how the use of AIT structures may vary with the task, environment, and other aspects that offer alternative sources of structures. One of the main sources of the structures within web-based IMS application are tasks and their types - to create technical or non-technical ideas/ to create textual, visual ideas. 'Webbased IMS task' is defined as the other source of structure in the AST framework. Based on the AST literature authors provide hypothesis: H2 Web-based IMS tasks influence web-based IM process and its outcomes. Qualitative metaanalysis supports this hypothesis. Some of possible variables described by researchers as 'web-based IMS task' are product idea, process idea (Bertetta 2015), problem decomposition, customized tasks (Luo, Tobia 2015), active IM, passive IM (Gamlin et al. 2007), improvement ideas (Deichmann 2012), Radical innovation ideas (Galbraith 1982). There are different ways how to research tasks. One way is to look at task focus as Gamlin et al. (2007) has proposed dividing all IM in two parts- passive that encourage people to submit all ideas that comes in mind (unfocused) and active- only focused ideas. Author also argues that active IM is more effective. Other way is to look at different feature 

adaptive structuration theory

of tasks, such as, Luo and Tobia (2015) has revealed that customized tasks and problem decomposition as task structures have very important role in IMS application. Third way is to research types of ideas that tasks are aimed to gain, for example, product/ process and improvement/radical ideas. Authors of this research advice is to select the most appropriate task evaluation criteria for the research context. Theoretical approach that is used in this research does not explore the all possible criteria of tasks. Because as practical examples have shown, that there could be textual and visual idea tasks. So, additional criteria could be type of task. More task criteria could have revealed in additional case study or web-based IMS data base research.

AST also refers to characteristics of group members and interactions within the group, such as members' knowledge and experience with AIT, agreement on appropriation, and styles of interacting. There are also other possible sources of structures such as an organization's environment where the artefact is embedded (Ajjan et al. 2016). In this paper this section will be used as characteristics of organization. As the group's internal system in AST, this construct can be defined as 'Organization internal system'. Based on the AST literature authors provide hypothesis: H3 Organization system influences web-based IMS application. Qualitative meta-analysis supports this hypothesis. Some of possible variables described by researchers are he repeated participation (previous experience) - the repeated participation in IMS using (Deichmann 2012; Bertetta 2015), leadership style, commitment to IMS (Deichmann 2012), in the idea network during IM process (Deichmann 2012; Bertetta 2015), network connectivity (ties) - idea generators network connectivity (Bjork, Magnusson 2009; Deichmann 2012), manager IM archetype (Vandenbosch et al. 2006), ideation capability (Lindross 2006),organisation innovation structure (Galbraith 1982).

Organisation system has the most possible research elements, that should be evaluated and chosen based on the research aim and context. For example, Vandenbosch et al. (2006) investigate the inter-relatedness of idea generation, problem solving and inquiry and propose IM archetypes that reflect managers' dominant approaches and a model of IM based on patterns of behaviour. And express that understanding of these types can improve management. That means that it is important part of organisation system. But Bakker et al. (2006) has noticed the importance of socialpolitical context of organisation. Not only network size (Deichmann 2012), but also its connectivity (Bjork, Magnusson 2009) influences IM. Tung et al. (2009) has express the importance of a customer-centric IM process, but Selart and Johansen (2011) - value-focused thinking by revealing that it facilitates ideation fluency, but also to constrain it. Van Dijk and van den Ende (2002) have created Creativity Transformation Model to clarify the organisational conditions for the successful use of suggestion systems (passive type of web-based IMS), that reflects some organisation system elements. These elements could be tested in the AST framework.

\section{Process}

The process consists of two main parts: social interaction or the application process and emergent sources of structures. Application process consists from two main sub-parts: appropriation of technology, decision making process (in this paper idea management process in web-based IMS). The appropriation of technology varies with organizations and levels. In this paper, is provided two level approach to appropriation - management and user. According to AST, appropriation improves the decision processes in the organization by structuration - act of bringing processes, resources, and other structures into appropriation. In this paper appropriation is defined as the extent to which structures in use are consistent or aligned with innovation spirit (DeSanctis, Poole 1994; Ajjan et al. 2016). In correspondence with the decision making in AST, the construct is defined as idea management process, based on the context. Overall social interaction construct in this paper is defined as 'web-based IMS application'. Based on the AST literature authors provide hypothesis: H4 Appropriation during IM influences dynamics of interactions. Qualitative meta-analysis supports this hypothesis. The variables could be appropriaton by managers and users.

Vandenbosh et al. (2006) has also expressed that managers IM archetype has important role in IM process that shows that not only users, but also managers have important role in IM process. So it is important to research not only appropriation of users, that is widely researched (e.g. Desanctis, Poole 1994; Ajjan et al. 2016). Because there will be no participative processes if the users or leaders does not wish to create/involve them (Dennis, Garfield 2003). Deichmann (2012) has admitted that a leader can influence the degree to which employees are committed to IM.

Emergent sources of structure will bring with it idea quality, idea quantity etc. These elements affect the success of web-based IMS adoption. As the emergent source of structure in AST, this construct is defined as 'Web-based IMS outputs in the processes. Based on the AST literature authors provide hypothesis: H5 IMS outputs in its application process influences the dynamics of the process and vice versa. Qualitative meta-analysis supports this hypothesis.

Most frequent researched IMS output variables are idea quantity and quality. Idea quality could be defined as the average quality of generated ideas (idea creativity) (Selart, Johansen 2011; MacCrimmon, Wagner 1994; Girotra et al. 2010; Deichmann 2012; Bjork, Magnusson 2009). There could be researched two hypotheses: (1) higher appropriation higher idea quality; (2) idea quality stimulates idea quantity. Idea quantity could be defined as number of ideas generated (MacCrimmon, Wagner 1994; Girotra et al. 2010; Deichmann 2012). Literature review develope two hypothesis: (1) higher appropriation, higher quantity; (2) idea quantity stimulates idea quantity.

Some of other possible variables described by researchers as 'web-based IMS outputs in the process' are variance in the quality, selected best ideas (Girotra et al. 2010), particiveness (Dennis, Garfield 2003), stimulus ideas (Luo, Tobia 2015), involvement productivity (Deichmann 2012). 

adaptive structuration theory

Idea quality and quantity are the most frequent applied measurements of IM and IMS, that shows that these are the main elements of web- based IMS application outputs. Denis and Garfield (2003) have revealed that GSS processes may encourage more participation that gives also challenge to research this element in IMS context. There can be also researched stimulus ideas impact on web-based IMS application and its results. Authors have noticed that there is no common view on IMS output elements, except idea quality and quantity. It would be advisable to create IMS effectiveness evaluation tool that would include most important output elements.

\section{Outputs and $O E$}

There are two main output variables in classical AST version - outcomes of the process and new social structures. Authors of this paper also include IMS process outputs in the output section.

In the AST framework, an organization's intention to apply web-based IMS is affected by the benefits of their application, for example, efficiency, quality, agreement, commitment, and other OE characteristics. Thus, this construct can be defined as 'Organizational effectiveness'. Based on the AST literature authors provide hypothesis: H6 Web-based IMS application is positively associated with OE. This hypothesis is supported by conducted research.

OE is a wide term. Research showed that in the OE literature 199 OE dimensions are mentioned. There are no right or wrong dimensions to research. All depends on research aims. In future research authors should select, which of these dimensions are important in certain contexts. There is also some evidence of OE dimensions in IM literature, for example Perez et al. (2013) has researched IMS within sustainability context, but Barczak et al. (2009) - new product development performance, Nilsson et al. (2002) - strengthen the innovation capability of an organisation. Boeddrich (2004) highlights benefits of internet-based system application - motivation increase, transparency increase, less conflict with patent-related problems.

Any new technology brings about a certain impact and causes certain problems that are determinant to raise of new social structures, such as rules and resources. The introduction of web-based IMS application could alter existing IM process, thereby causing a potential change in the organizational structure. As a new social structure in AST, this construct is defined as 'New structures derived from web-based IMS application'. Based on the AST literature authors provide hypothesis: H7 Process can result in new structures derived from web-based IMS application.

Qualitative meta-analysis supports this hypothesis. For example, Bertetta (2015) has noticed that web-based IMS application could create new challenges for managers. One of the problems of IMS that occurs after their application process is the difficulty to accurately assess ideas (Westerski 2013; Westerski et al. 2013; Perez et al. 2013). Employed idea assessment method in web-based IMS is simple idea, evaluation methods most often being analysis of community statistics (number of ideas per user, community voting results, number of idea comments etc.) or internal business metrics that are delivered by designated experts (e.g. return of investment and market value). Westerski et al. (2013) have even developed idea taxonomy that could improve idea assessment. That shows that this and other challenges that occur in IMS application could be the determinant for development of new structures.

DeSanctis and Poole (1994) also suggest that analysis based on AST can identify certain patterns more likely associated with OE than others (Maznevski, Chudoba 2000), that shows that it is possible to research also how webbased IMS outputs influence OE. Authors provide hypothesis that IMS outputs influences OE. Or in other words IMS outputs mediate between web-based IMS application and OE. This hypothesis is not directly supported by literature review, but it should be tested, to explore if IMS outputs could be predictor of OE: H8 IMS outputs influences OE.

\section{Conclusions and discussion}

The research based on the literature review provides understanding how to conceptualize web-based IMS application and OE by applying AST. It proposes a research framework based on AST to describe the web-based IMS and OE relations. There is also proposed 8 hypothesis that developed based on the literature review and analysed with qualitative meta-analysis.

\section{Implications}

This paper offers several theoretical implications for scholars and researchers. First, the AST has been used in variety of research contexts, but it has not been extensively applied to domain of web-based IMS. This study provides useful framework for the assessment of web-based IMS and OE relations within AST context and detailed characterization of AST construct in web-based IMS application context. The study creates research framework to research AST not focusing on groups or individuals, but on organizations. It expands the domain of outcomes of AST researching outcomes of web-based IMS by including OE dimensions that are normally found in the OE literature as possible outcomes of web-based IMS application. Paper extend the AST output dimensions by suggesting 199 possible OE dimensions to be applied according to the context and research aim. The study also suggests exploring web-based IMS application process outcomes as determinant of OE. Paper generates an in-depth understanding of main factors and processes associated with application of web-based IMS.

Second, results provide some insights that may help in designing future studies. They highlight the importance of empirical and theoretical research to select elements to include in AST framework to research web-based IMS. It also demonstrates that there are a lot of possible variables to research. In future studies researchers should evaluate and 

adaptive structuration theory

select the most appropriate variables for focused studies. The framework suggests that web-based IMS application depends on technology structures, tasks structures, organization system, appropriation, web-based IMS outputs that results in $\mathrm{OE}$ and new structures.

\section{Limitations and future studies}

There have been two main limitations: (1) analyzed literature sources amount based on the research design (selected data bases, time frame and selection approach); (2) theoretical approach. Applied approach has limited specified general model development and led to the many possible research dimensions that are only theory based. For example, web-based IMS technological features are explored only based on theory. So, it reflects only limited aspects of it. Based on the limitations authors have develop suggestions for future studies. Additional research should be conducted to explore basic web-based IMS features based on empirical research. There have been researches conducted by the paper authors that showed that there are 20 technological features that are important to assess while choosing webbased IMS. Features are described only based on technology overview but not evaluated by the experts and practitioners. It would be advisable to mix methods in the future to research technology feature element. That is, conduct technology research by gathering the most frequent applied features in technologies and expert method to pick the most important ones and to verify results.

For future studies, the most appropriate elements should be selected in each of the sections, based on the research aim and focus. For example, organisation system has the most possible research elements, that should be evaluated and chosen based on the research aim and context. Authors have noticed that there is no common view on IMS output elements, except idea quality and quantity. It would be advisable to create IMS effectiveness evaluation tool that would include the most important output elements. It should be also expressed, that in this general framework only basic hypothesis are developed. For different research aims the research variables for all hypotheses could variate.

Because paper length limitations did not allow to represent full research, developed sub-hypotheses and content analysis (frequently applied AST research methods etc.) results are not fully included. The general framework also includes only elements and possible variables that are found in IM and IMS literature, but possible variables based on AST literature should be overviewed as well. For example, in the future the agents' role should be much more emphasized in the IMS context and it should be explained in more detail how agents produce new structures.

There could be a critique that Giddens intended Structuration theory to be abstract and theoretical, not practical. That is the reason why authors based this article on Poole and DeSanctis who developed AST is more detailed, practical and suitable for advanced information technology context. In AST researches different methodologies are approbated that have shown how to research advanced information technology influence on outputs and outcomes. In this paper outcomes are defined as web-based IMS outcomes, but outputs as OE, so showing the trend in the literature that different OE metrics should be researched. It is not dubious task to relate constructively between IMS and OE if the right methodology and metrics are applied. In future study authors plan to attract experts to validate all potential variables and range them for more specific model and evaluate the most frequent applied AST research methods. Experts will not only be AST and IMS researchers, but also IMS professionals to balance theoretical and practical views. Authors hope that this paper will stimulate scientific discussion and further researches about web-based IMS application impact on OE.

\section{References}

Ajjan, H.; Kumar, R. L.; Subramaniam, C. 2016. Information technology portfolio management implementation: a case study, Journal of Enterprise Information Management 29(6): 841-859. https://doi.org/10.1108/JEIM-07-2015-0065

Angle, H.; Perry, J. L. 1981. An empirical assessment of organizational commitment and organizational effectiveness, Administrative Science Quarterly 26(1): 1-14. https://doi.org/10.2307/2392596

Applegate, L. M. 1986. Idea management in organization planning (brainstorming, strategy): Dissertation thesis. University of Arizona.

Bakker, H.; Boersma, K.; Oreel, S. 2006. Creativity (ideas) management in industrial r\&d organizations: a crea- political process model and an empirical illustration of corus RD\&T, Creativity and Innovation Management 15(3): 296-309. https://doi.org/10.1111/j.1467-8691.2006.00397.x

Barczak, G.; Griffin, A.; Kahn, B. K. 2009. PERSPECTIVE: trends and drivers of success in npd practices: results of the 2003 PDMA best practices study, Journal of Product Innovation Management 26(1): 3-23. https://doi.org/10.1111/j.15405885.2009.00331.x

Bertetta, M. 2015. The role of idea management systems for innovation in large organizations: 3 essays: Dissertation thesis. School of Business and Social Sciences.

Biswas, S. 2010. Relationship between psychological climate and turnover intentions and its impact on organisational effectiveness: a study in Indian organisations, IIMB Management Review 22(1): 102-110. https://doi.org/10.1016/j.iimb.2010.04.013

Bjork, J.; Magnusson, M. 2009. Where do good innovation ideas come from? Exploring the influence of network connectivity on innovation idea quality, Journal of Product Innovation Management 26(1): 662-670. https://doi.org/10.1111/j.15405885.2009.00691.x 
Boeddrich, H. J. 2004. Ideas in the workplace: a new approach towards organizing the fuzzy front end of the innovation process, Creativity and Innovation Management 13(4): 274-285. https://doi.org/10.1111/j.0963-1690.2004.00316.x

Boiral, O. 2012. ISO 9000 and organizational effectiveness: a systematic review, QMJ 19(3): 16-37.

Bose, U. 2015. Design and evaluation of a group support system supported process to resolve cognitive conflicts, Computers in Human Behavior 49(1): 303-312. https://doi.org/10.1016/j.chb.2015.03.014

Bothos, E.; Apostoulou, D.; Mentzas, G. 2012. Collective intelligence with web-based information aggregation markets: the role of market facilitation in idea management, Experts Systems with Applications 39(1): 1333-1345. https://doi.org/10.1016/j.eswa.2011.08.014

Charlier, S. D.; Stewart, G. L.; Greco, L. M.; Reeves, C. J. 2016. Emergent leadership in virtual teams: a multilevel investigation of individual communication and team dispersion antecedents, The Leadership Quarterly 27(5): 745-764. https://doi.org/10.1016/j.leaqua.2016.05.002

Deichmann, D. 2012. Idea management: perspectives from leadership, learning, and network theory: Dissertation thesis. ERIM.

Deng, X.; Joshi, K. D.; Galliers, R. D. 2016. The duality of empowerment and marginalization in microtask crowdsourcing: giving voice to the less powerful through value sensitive design, MIS Quarterly 40(2): 279-322.

Dennis, A. L.; Garfield, M. J. 2003. The Adoption and Use of GSS in project teams: toward more participative processes and outcomes, MIS Quarterly 27(2): 289-323.

Dension, D. R. 1990. Corporate culture and organizational effectiveness. New York: John Wiley \& Sons.

DeSanctis, G.; Poole, M. S. 1994. Capturing the complexity in advanced technology use: adaptive structuration theory, Organization Science 5(2): 121-147. https://doi.org/10.1287/orsc.5.2.121

Diaz Andrade, A.; Doolin, B. 2016. Information and communication technology and the social inclusion of refugees, MIS Quarterly 40(2): 405-416.

Droge, C.; Vickery, S. K.; Jacobs, M. A. 2012. Does supply chain integration mediate the relationships between product/process strategy and service performance? An empirical study, International Journal Production Economics 137(1): $250-262$. https://doi.org/10.1016/j.ijpe.2012.02.005

Edgington, T. M.; Raghu, T. S.; Vinze, A. S. 2010. Using process mining to identify coordination patterns in IT service management, Decision Support Systems 49(1): 175-186. https://doi.org/10.1016/j.dss.2010.02.003

Galbraith, J. R. 1982, Designing the innovating organization, Organizational Dynamics 10(3): 5-25. https://doi.org/10.1016/00902616(82)90033-X

Gamlin, J. N.; Yourd, R.; Patric, V. 2007. Unlock creativity with “active" idea management, Research Technology Management 50(1): 13-16.

Ganju, K. K.; Pavlou, P. A.; Banker, R. D. 2016. Does information and communication technology lead to the well-being of nations? A country-level empirical investigation, MIS Quarterly 40(2): 417-441.

Giddens, A. 1984. The constitution of society. New York: Basic Books.

Girotra, K.; Terwiesch, C.; Ulrich, K. T. 2010. Idea generation and the quality of the best idea, Management Science 56(4): 591 605. https://doi.org/10.1287/mnsc.1090.114

Goodman, P. S.; Saks, A. M. 1977. New perspectives on organizational effectiveness. San Francisko: Jossey - Bass.

Gopal, A.; Bostrom, R. P.; Chin, W. W. 1992/1993. Applying adaptive structuration theory to investigate the process of group support systems use, Journal of Management Information Systems 9(3): 45-69. https://doi.org/10.1080/07421222.1992.11517967

Hyun-Kim, S.; Mukhopadhyay, T.; Kraut, R. E. 2016. When does repository kms use lift performance? The role of alternative knowledge sources and task environments, MIS Quarterly 40(1): 133-164.

Hooff, B.; Groot, J.; Jonge, S. 2005. Situational influences on the use of communication technologies: a meta-analysis and exploratory study, Journal of Business Communication 42(1): 4-27. https://doi.org/10.1177/0021943604271192

Iyengar, K.; Sweeney, J. R; Montealegre, R. 2015. Information technology use as a learning mechanism: the impact of it use on knowledge transfer effectiveness, absorptive capacity, and franchisee performance, MIS Quarterly 39(3): 615-648.

Im, I.; Jun, J.; Oh, W.; Jeong, S. O. 2016. Deal-seeking versus brand-seeking: search behaviors and purchase propensities in sponsored search platforms, MIS Quarterly 40(1): 187-204.

INSEAD. 2016. The global innovation index 2016 [online], [cited 14 January 2017]. Available from Internet: www.globalinnovationindex.org/gii-2016-report\#

Kang, D.; Santhanam, R. 2003/2004. A longitudinal field study of training practices in a collaborative application environment, Journal of Management Information Systems 20(3): 257-281.

Kessler, E. H. 2013. Encyclopedia of management theory. Newbury Park: SAGE Publications. https://doi.org/10.4135/9781452276090

Ketter, W.; Peters, M.; Collins, J.; Gupta, A. 2016. A multiagent competitive gaming platform to address societal challenges, MIS Quarterly 40(2): 447-460.

Kirkman, B. L.; Mathieu, J. E. 2005. The dimensions and antecedents of team virtuality, Journal of Management 31(5): 700-718. https://doi.org/10.1177/0149206305279113

Lecy, J. D.; Scmitz, H. P.; Swedlund, H. 2012. Non-govermental and not-for-profit organizational effectiveness: a modern synthesis, Voluntantas: International Journal of Voluntary and Nonprofit Organizations 23(2): $434-457$. https://doi.org/10.1007/s11266-011-9204-6

Lee, H. K.; Zo, H. 2016. Assimilation of military group decision support systems in Korea: the mediating role of structural appropriation, Information Development 33(1): 14-28. https://doi.org/10.1177/0266666916628316 

adaptive structuration theory

Lee, L. W. 2012. Group decision making with incomplete fuzzy preference relations based on the additive consistency and the order consistency, Expert Systems with Applications 39(4): 11666-11676. https://doi.org/10.1016/j.eswa.2012.04.043

Lin, C. I. C.; Myers, M. D. 2015. Extending Ict4d studies: the value of critical research, MIS Quarterly 39(3): 697-712.

Lindross, M. 2006. Developing Front-End idea management and information processing. Working paper. Innovation Management Institute, Helsinki University of Technology.

Lolli, F.; Ishizaka, A.; Gamberini, R.; Rimini, B.; Messori, M. 2015. FlowSort-GDSS - a novel group multi-criteria decision support system for sorting problems with application to FMEA, Expert Systems with Applications 42(17/18): 6342-6349. https://doi.org/10.1016/j.eswa.2015.04.028

Lowe, M.; Heller, J. E. 2014. PLM reference model for integrated idea and innovation management, Product Lifecycle Management for a Global Market, IFIP Advances in Information and Communication Technology 442(1): 257-266.

Luo, L.; Toubia, O. 2015. Improving online idea generation platforms and customizing the task structure on the basis of consumers' domain-specific knowledge, Journal of Marketing 79(5): 100-114. https://doi.org/10.1509/jm.13.0212

MacCrimmon, K. R.; Wagner, C. 1994. Stimulating ideas through creative software, Management Science 40(11): $1514-1532$. https://doi.org/10.1287/mnsc.40.11.1514

Mausolff, C.; Spence, J. 2008. Performance measurement and program effectiveness: a structural equation modeling approach, International Journal of Public Administration 31(6): 595-615.

Maznevski, M. M.; Chudoba, K. M. 2000. Bridging space over time: global virtual team dynamics and effectiveness, Organization Science 11(5): 473-492. https://doi.org/10.1287/orsc.11.5.473.15200

Mikelsone, E.; Liela, E. 2015. Discussion on the terms of idea management and idea management systems, Journal of Regional Formation and Development Studies 3(17): 97-110.

Mikelsone, E.; Liela, E. 2016. Idea management and organizational effectiveness: research gap, Journal of Business Management 12(1): 4-24.

Mutch, A. 2002. Actors and networks or agents and structures: towards a realist view of information system, Organisation 9(3): 477-498. https://doi.org/10.1177/135050840293013

Nilsson, L.; Elg, M.; Bergman, B. 2002. Managing ideas for the development of new products, International Journal of Technology Management 24(5/6): 498-513. https://doi.org/10.1504/IJTM.2002.003067

Perez, A.; Larrinaga, F.; Curry, E. 2013. The role of linked data and semantic-technologies for sustainability idea management, Chapter in Counsell, S. (Ed.). Software Engineering and Formal Methods. Berlin: Springer-Verlag, 306-312.

Poole, M. S. 2013. Adaptive structuration theory. Chapter in Hessler, E. H. (Ed.). Encyclopedia of management theory. Newbury Park: SAGE Publications, 22-25. https://doi.org/10.4135/9781452276090.n7

Poole, M. S.; DeSanctis, G. 1990. Undrstanding the use of group decision support systems: the theory of adaptive structuration. Chapter in Steinfied, C. W.; Fulk, J. (Eds.). Organizations and Communication Technology. Newbury Park: SAGE Publications, 173-193.

Reinig, B. A; Shin, B. 2002. The dynamic effects of group support systems on group meetings, Journal of Management Information Systems 19(2): 303-325.

Sabherwal, R.; Jeyaraj, A. 2015. Information technology impacts on firm performance: an extension of Kohli and Devaraj (2003), MIS Quarterly 39(4): 809-836.

Saskia-Bayerl, P.; Lauche, K.; Axtell, C. 2016. Revisiting group-based technology adoption as a dynamic process: the role of changing attitude-rationale configurations, MIS Quarterly 40(3): 775-795.

Schmitz, K. W.; Teng, J. T. C.; Webb, K. J. 2016. Capturing the Complexity of Malleable IT Use: Adaptive Structuration Theory for individuals, MIS Quarterly 40(3): 663-686.

Selart, M.; Johansen, S. T. 2011. Understanding the role of value - focused thinking in idea management, Creativity and Innovation Management 20(3): 196-206. https://doi.org/10.1111/j.1467-8691.2011.00602.x

Summa, A. 2004. Software tools to support innovation process- focuss on idea management. Working paper [29]. Innovation Management Institute, Helsinki University of Technology.

Tung, W. F.; Yuan, S. T.; Tsai, J. R. 2009. A custom collaboration service system for idea management of mobile phone design, Human Factors and Ergonomics in Manufacturing 19(5): 494-509. https://doi.org/10.1002/hfm.20147

Vaismoradi, M.; Turunen, H.; Bondas, T. 2013. Content analysis and thematic analysis: Implications for conducting a qualitative descriptive study, Nursing \& Health Sciences 15(3): 398-405. https://doi.org/10.1111/nhs.12048

Van Dijk, C.; Van Den Ende, J. 2002. Suggestion systems: transferring employee creativity into practicable ideas, R\&D Management 32(5): 387-395. https://doi.org/10.1111/1467-9310.00270

Vandenbosch, B.; Saatcioglu, A.; Fay, S. 2006. Idea management: a systematic view, Journal of Management Studies 43(2): 259288. https://doi.org/10.1111/j.1467-6486.2006.00590.x

Wang, D.; Xiang, Z.; Fesenmaier, D. R. 2016. Smartphone use in everyday life and travel, Journal of Travel Research 55(1): 5263. https://doi.org/10.1177/0047287514535847

Westerski, A. 2013. Semantic technologies in idea management systems: a model for interoperability, linking and filtering: Dissertation thesis. Universidad Politecnica de Madrid.

Westerski, A.; Dalamagas, T.; Iglesias, C. A. 2013. Classifying and comparing community innovation in Idea Management Systems, Decision Support Systems 54(1): 1316-1326. https://doi.org/10.1016/j.dss.2012.12.004

Wiener, M.; Mahring, M.; Remus, U.; Saunders, C. 2016. Control configuration and control enactment in information systems projects: review and expanded theoretical framework, MIS Quarterly 40(3): 741-789. 